\title{
Effects of caudate and cortical lesions on place and response learning in rats
}

\author{
W. GARY THOMPSON, MARSHA O. GUILFORD, and LESLIE H. HICKS \\ Howard University, Washington, D.C. 20059
}

\begin{abstract}
Rats with lesions in the caudate nucleus made fewer errors and took fewer trials to reach criterion than unoperated rats in acquisition and reversal performance on a T-maze task. On place and response performance, caudate and cortical rats did not differ from unoperated rats in number of errors and trials to criterion. Caudate rats were predominantly place learners, while cortical rats were mainly response learners. The results are discussed in relation to compensatory behavioral mechanisms following brain lesions.
\end{abstract}

Several experiments show that rats, in a simple T-maze, can use spatial or kinesthetic cues in learning place or response habits (Blodgett \& McCutchan, 1947; Ritchie, Aeschliman, \& Peirce, 1950; Tolman, Ritchie, \& Kalish, 1947). Although Restle (1957) has made a behavioral analysis of the place vs. response controversy, there have been no studies directly assessing the neural bases of place and response performance. Potegal's (1969) report implies that the brain structures involved in place learning are different from those involved in response learning. He found that caudate nucleus damage prevented rats from learning the position of a food reward in a radial maze when the reward was placed in a constant direction relative to the animal's body position. Potegal suggested that the caudate nucleus functioned as a system for spatial localization. Positions in space were defined by their distance and direction from an observer, for example " 1 meter to its left." In a later review, Potegal (1972) referred to some of his unpublished data which show that caudate lesions interfere with response learning but not place learning. He states that response learning reflects the operation of this caudate spatial localization system.

Further evidence of specific neural involvement in a similar system of spatial localization is provided by Pohl (1973). He found that damage to the frontal cortex produced a marked performance deficit when monkeys were required to displace an object that was always on their right side. Animals with parietal lesions were not impaired on this task. But, on a spatial discrimination task, using an external rather than a body position referent, parietal animals were impaired and frontal animals were not.

The anatomical projections of the cerebral cortex to the caudate nucleus have led to a search for regional specificity in the caudate, although histologically this

This research was supported by Research Grant 1 R01 NS 09630 to L.H.H. and was completed during his stay at the Center for Advanced Study in Behavioral Sciences, Stanford, California. Address reprint requests to Leslie H. Hicks, Psychology Department, Howard University, Washington, D.C. 20059. nucleus gives an appearance of uniformity (for a review, see Divac, 1971). Rosvold (1968, p. 453) states that, in the monkey, "there seems to be no exception, then, to the rule that whenever a frontal lesion results in impaired performance on a test, so do lesions in the head of the caudate." Similar findings have been reported in the rat (Divac, 1971; Hannon \& Bader, 1974; Kolb, 1977).

Divac, Rosvold, and Szwarcbart (1967) found that lesions in the tail of the caudate impaired performance by monkeys on visual discrimination tasks, as did ablation of the cortical area projecting to the caudate tail (the inferotemporal cortex). Medial frontal cortical ablations or lesions in that part of the caudate to which this cortical region projects produced impairment on a spatial reversal task in rats (Divac, 1971). Livesey and Muter (1976) found that stimulation that blocked functioning of the anterodorsal caudate of rats produced deficits on spatial reversal but not on form discrimination, while stimulation to the posteroventral caudate impaired form discrimination reversal but not spatial reversal. Kirkby (1969) also demonstrated this reversal deficit. Caudate animals performed as well as unoperated controls in the acquisition of a brightness discrimination in a Y-maze. On the reversal learning of this problem, caudate animals were impaired compared with cortical and unoperated subjects. Kirkby attributed this deficit in the caudate performance to an "inability to inhibit the initially trained response."

The present experiments were designed to provide further information on the role of the cortex and caudate nucleus in kinesthetic and spatial learning.

\section{GENERAL METHODS}

\footnotetext{
Subjects

One hundred and eight male albino rats (Sprague-Dawley, Madison, Wisconsin) were used. The subjects were approximately 100 to 130 days old at the start of the experiments. They were housed individually and maintained on a 12-h light/12-h dark cycle. Rats were assigned randomly to the experimental groups in each experiment.
} 


\section{Surgery and Histology}

Surgery was done while the rat was anesthetized from an intraperitoneal injection of Nembutal $(50 \mathrm{mg} / \mathrm{kg}$ of body weight). The caudate animals received lesions at sites determined by stereotaxic coordinates based on the atlas of Pellegrino and Cushman (1967). Caudate lesions were produced by a .25 -mm-diam stainless steel electrode with a bare tip of $.5 \mathrm{~mm}$. Lesions in the cortical animals were produced by aspiration techniques. Both caudate and cortical lesions were bilateral.

When each experiment was completed, the operated animals were anesthetized and perfused through the heart with sterile saline followed by a $10 \%$ Formalin solution. The brains from all operated animals were cut into sections of $40 \mu$, and every 10th section was stained with cresyl violet solution.

\section{EXPERIMENT 1}

This experiment investigated the effects of lesions of the cortex and the caudate on the acquisition and reversal of place and response learning by rats. If the hypothesis of Potegal (1972) is correct, animals in our caudate group should be place, rather than response, learners. Use of response cues, which are necessary for spatial localization with a body reference, should be impaired by caudate damage. Pohl's (1973) conclusion that parietal lesions interfere with performance based on external cues implies that the animals in our cortical group, in which the damaged area is parietal, should be response, rather than place, learners. Our reversal task tests the hypothesis of Kirkby (1969) that caudate animals perform poorly because of an inability to inhibit previously learned responses. Both Hicks (1964) and Mackintosh (1965) found that overtraining increased the number of response learners in a maze task that could be learned on the basis of either place or response habits. Overtraining was varied in the present experiment to determine its effect on the performance of the animals with lesions.

\section{Method}

Subjects. Thirty-six rats were assigned randomly to 3 groups of 12 each: caudate, cortex, and unoperated controls.

Surgery. Coordinates for the caudate lesions were $1.4 \mathrm{~mm}$ anterior to bregma, $3.4 \mathrm{~mm}$ lateral to the midline suture, and $5 \mathrm{~mm}$ below the surface of the skull. An anodal current of $2 \mathrm{~mA}$ was passed through the electrode for $10 \mathrm{sec}$. The cortical lesion site was also determined stereotaxically and its boundaries were determined by the following coordinates: anterior +3.2 to posterior -2.2 , lateral \pm 3.5 , and height $2.0 \mathrm{~mm}$. A metal L-shaped aspiration pipette, with an outer diameter of $3.0 \mathrm{~mm}$ and under $7 \mathrm{lbs}$ of negative pressure for $15 \mathrm{sec}$, was used to produce the cortical lesions.

Apparatus. The apparatus was a double T-maze built in the shape of a plus sign. At the ends of the north and south arms were startboxes; goalboxes were at the ends of the east and west arms. Each maze arm was $121 \mathrm{~cm}$ long, the width was $19.4 \mathrm{~cm}$, and the height was $41 \mathrm{~cm}$. The entire wooden maze was painted a neutral gray. The maze was placed on a table in a room with fluorescent light. White noise was used to mask extraneous sounds.

Pretraining. All the subjects were maintained on a 23-h fooddeprivation schedule. Water was always available in the home cage. There were 5 days of maze adaptation during which the subject was allowed to explore the maze freely for $15 \mathrm{~min}$. All arms of the maze, both goalboxes, and the startboxes were open for exploration. Food pellets $(45 \mathrm{mg}, \mathrm{P}$. J. Noyes Co.) were in both goalboxes during the adaptation session. On the fifth day of adaptation, a preference test was run. Seven trials from the south startbox were given. The north startbox and arm were closed off so that the maze was now in a T-configuration. The subject could only turn right or left at the choice point. The preference criterion was four turns in the same direction. No food was in the goalboxes, and the food containers were placed face down during these preference trials.

Acquisition. The subjects were trained to run to their nonpreferred side. All the subjects were run from the south startbox and received 20 trials per day until 10 consecutive correct responses were made. On correct responses, the animal was allowed to remain in the goalbox until it ingested the reward pellets; it was then returned to the startbox. On incorrect responses, the subject was removed immediately from the goalbox and returned to the startbox. The interval in the startbox between trials was $20 \mathrm{sec}$.

Test trial. The subjects received the test trial immediately following the attainment of the acquisition criterion. The test trial was conducted from the startbox opposite to that used during acquisition, that is, the north rather than the south startbox. No pellets were in the goalboxes. If the subject went to the same location where reward was previously received (and therefore turned in the opposite direction), it was labeled a place learner. If the subject turned in the same direction as it did during acquisition and went to a different place, it was scored as a response learner.

Reversal training. On the day following the test trial, reversal training was begun. Place learners were trained on response and response learners were trained on place. Both north and south startboxes were used during reversal training, and 10 trials were run daily from each direction. These 20 trials a day were given until a criterion of 10 consecutive correct responses was reached.

\section{Results}

Histology. The location of lesions is shown in representative reproduction in Figure 1. The caudate lesions were small and located mostly in the center of the nucleus, but some were dorsal and a few were

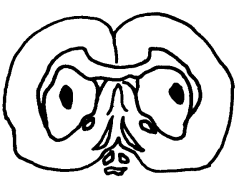

3.0

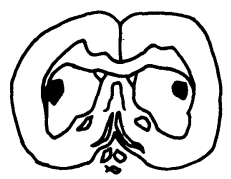

2.6

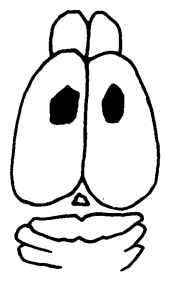

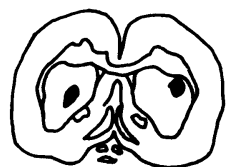

2.2

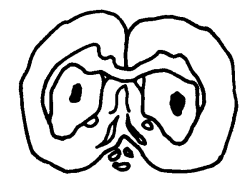

2.2

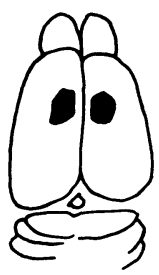

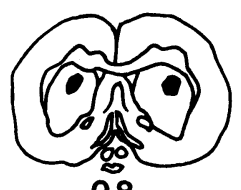

0.8

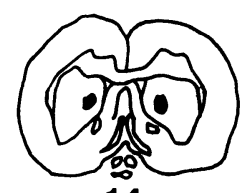

1.4

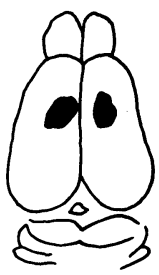

Figure 1. Representative caudate and cortical lesions. 

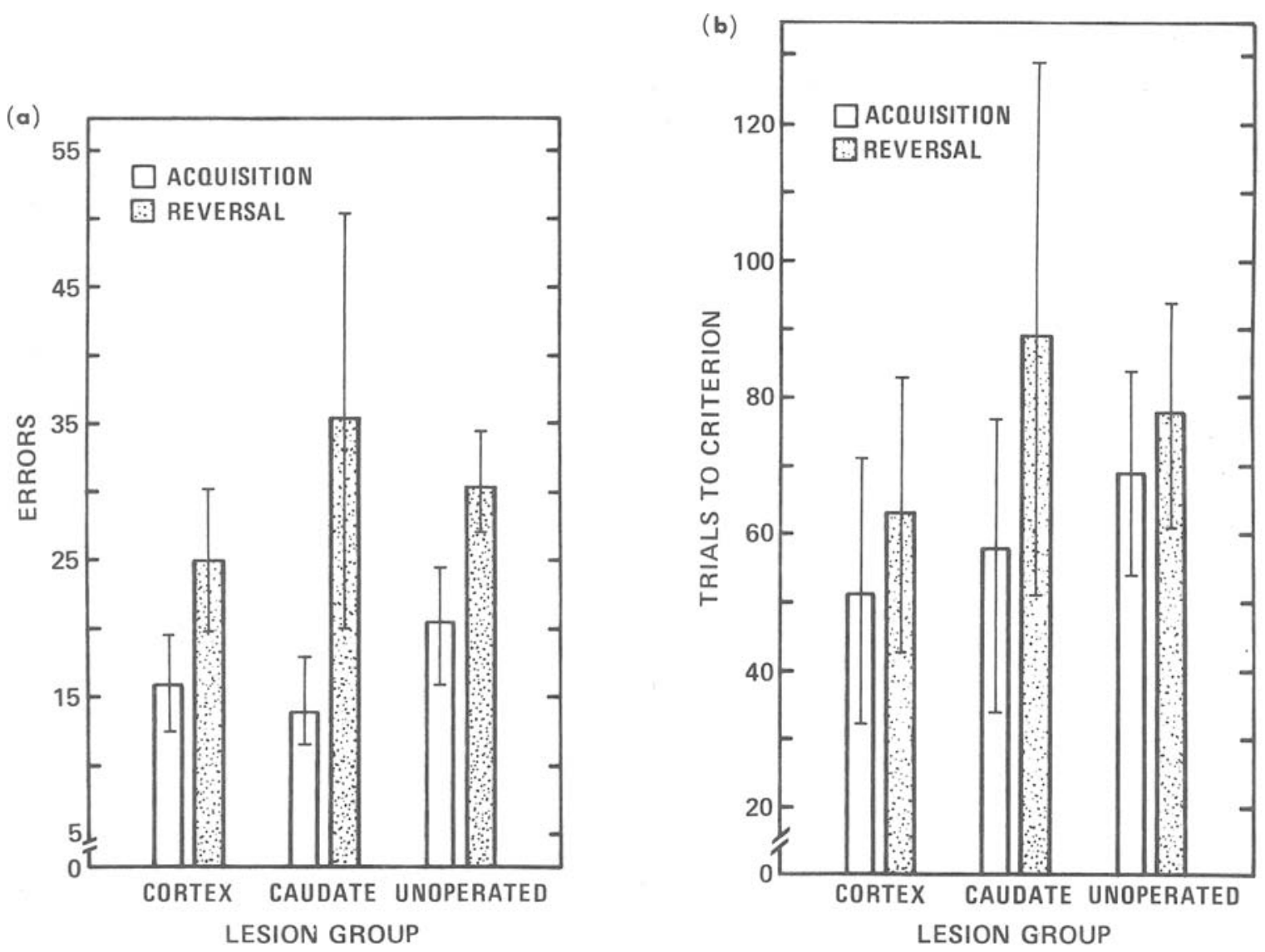

Figure 2. (a and b) Means and standard deviations for acquisition error and trials-to-criterion scores.

mainly ventral. Their anterior-posterior extent was from 6.8 to $9.0 \mathrm{~mm}$ (based on the atlas of Pellegrino \& Cushman, 1967). The cortical lesions were also small and confined to areas 3, 4, 7, and 1 (Krieg, 1946). Subcortical tissue was not invaded surgically.

Acquisition. The means and standard deviations for errors and trials to criterion are shown in Figures $2 \mathrm{a}$ and $2 \mathrm{~b}$. On neither of these sets of acquisition scores did an analysis of variance show a difference among the groups $[F(2,33)<1.0]$.

The time taken by the animal to run from the start to the goalboxes was also measured, and the median scores for the various groups were: cortex, $59.10 \mathrm{sec}$; caudate, $20.67 \mathrm{sec}$; unoperated, $11.01 \mathrm{sec}$. An analysis of variance showed that the running time scores were significantly different among the various groups $[F(2,33)=5.46, p<.01]$.

Test trial. The number of place and response learners based on performance on the test trial is shown in Table 1. The frequencies of place and response learners were analyzed by a chi-square test, which showed that the differences among the groups were significant $\left[\chi^{2}(5)=6.34, p<: 01\right]$. More of the caudate groups were place learners than response learners and more of the cortex group were response learners than place learners. Table 1 also shows that overtraining had no effect on the frequency of place or response learners in any of the groups.

Reversal. The mean number of trials to criterion, errors, and their standard deviations are displayed in Figures $2 \mathrm{a}$ and $2 \mathrm{~b}$. Analysis of variance revealed no difference between acquisition and reversal trials to criterion, but significantly more errors were made in the reversal than in the acquisition phase of the experiment $[F(1,33)=8.21, p<.01]$. This effect was attributable to the caudate group, which was the only one with a significant difference between acquisition and reversal errors $[\mathrm{t}(11)=3.52, \mathrm{p}<.01]$. There were no significant differences among the groups on reversal

Table 1

Number of Place (P) and Response (R) Learners in the Various Groups as a Function of Overtraining

\begin{tabular}{|c|c|c|c|c|c|c|}
\hline \multirow{2}{*}{$\begin{array}{l}\text { Overtrain- } \\
\text { ing }\end{array}$} & \multicolumn{2}{|c|}{ Cortex } & \multicolumn{2}{|c|}{ Caudate } & \multicolumn{2}{|c|}{ Nonoperated } \\
\hline & $\mathbf{P}$ & $\mathbf{R}$ & $\mathbf{P}$ & $\mathbf{R}$ & $\mathbf{P}$ & $\mathbf{R}$ \\
\hline 0 & 2 & 4 & 5 & 1 & 3 & 3 \\
\hline 100 & 2 & 4 & 5 & 1 & 3 & 3 \\
\hline
\end{tabular}


trials to criterion or error scores $(F<1.0)$, and overtraining did not affect reversal scores $(F<1.0)$. There were also no significant differences in response times among the groups on the reversal phase of the $\operatorname{task}[\mathrm{F}(2,33)=2.81, \mathrm{p}>.05]$.

\section{EXPERIMENT 2}

Because the caudate nucleus has been shown to have specific anatomical regions associated with different behavioral functions (Divac et al., 1967; Livesey \& Muter, 1976; Neill, Boggan, \& Grossman, 1974; Winocur, 1974), a maze reversal task with different sensory cues was used to assess the effects of lesions in different regions of the caudate. The anterior caudate appears to be involved in spatial reversal performance (Divac, 1971; Hannon \& Bader, 1974; Kolb, 1977), while the posterior caudate is involved in performance on visual discrimination tasks (Divac et al., 1967). Mikulas (1966) showed that a deficit in spatial alternation performance produced by lesions in the head of the caudate in rats could be reduced by the presence of a light at the choice point indicating the correct arm. The light cue had a slight disruptive effect on unoperated rats.

The aim of the study was to determine whether cues relevant to the behavioral task would minimize the impairments that typically result from lesions to the anterior or posterior caudate. If the anterior caudate is more involved in mediating kinesthetic information and the posterior caudate is more involved in visual performance, then the addition of tactile and visual cues should affect the performance of animals with lesions in these caudate regions differently. In a task that can be learned by response (kinesthetic) or place (visual) habits, the performance of rats with lesions in the anterior caudate should be better in the presence of additional visual cues, since the damaged anterior caudate would not mediate tactile cues as effectively as a normal caudate. By the same reasoning, the posterior caudate animals might be expected to do worse when additional visual, rather than tactile, cues are present.

\section{Method}

Subjects. Seventy-two rats were assigned randomly to one of the following groups $(n=8$ per group): anterior caudate, no additional cues; anterior caudate, tactile cues; anterior caudate, visual cues; posterior caudate, no additional cues; posterior caudate, tactile cues; posterior caudate visual cues; nonoperated controls, no additional cues; nonoperated controls, visual cues.

Surgery. The stereotaxic coordinates (with bregma as a reference) were as follows: anterior caudate $(\mathrm{A}+2.6, \mathrm{~L} \pm 3.0, \mathrm{H}-5.5)$; posterior caudate $(\mathrm{P}-.4, \mathrm{~L} \pm 5.0, \mathrm{H}-6.5)$. To produce the lesions in the anterior caudate, an anodal current of $2 \mathrm{~mA}$ was passed through the electrode for $20 \mathrm{sec}$. A current of $1 \mathrm{~mA}$ for $15 \mathrm{sec}$ was used for lesions in the posterior caudate.

Apparatus. The apparatus, pretraining, and acquisition procedures described in Experiment 1 were used in this experiment. But in this experiment, the reversal task involved training to the previously nonrewarded arm of the T-maze, and all trials were run from the same startbox as in acquisition. Reversal training began on the day after an animal had achieved 10 consecutive correct responses on acquisition.

For those animals in the tactile cue groups, coarse sandpaper (painted the same color of gray as the maze) covered the floor of the arm that led to the rewarded goalbox. In the visual cue condition, a mat board $(10 \times 25 \mathrm{~cm})$, with black vertical lines on one-half side and black horizontal lines on the other (the lines were $1.27 \mathrm{~cm}$ wide and $1.27 \mathrm{~cm}$ apart), was placed on the maze wall at the choice point. Both patterns were visible before the animal reached the choice point.

\section{Results}

Histology. Figures $3 \mathrm{a}$ and $3 \mathrm{~b}$ present reconstructions of typical anterior and posterior caudate lesions, respectively. Anterior lesions generally involved the middle and lateral regions of the caudate. There was occasional damage to the overlying cortex and the corpus callosum.

The posterior caudate lesions generally damaged the ventral aspects of the caudate. There was also damage in some animals to adjacent cortical and callosal tissue. Other structures occasionally damaged by lesions intended for the posterior caudate were amygdala, claustrum, and hippocampus. But involvement of these structures was slight, and none were

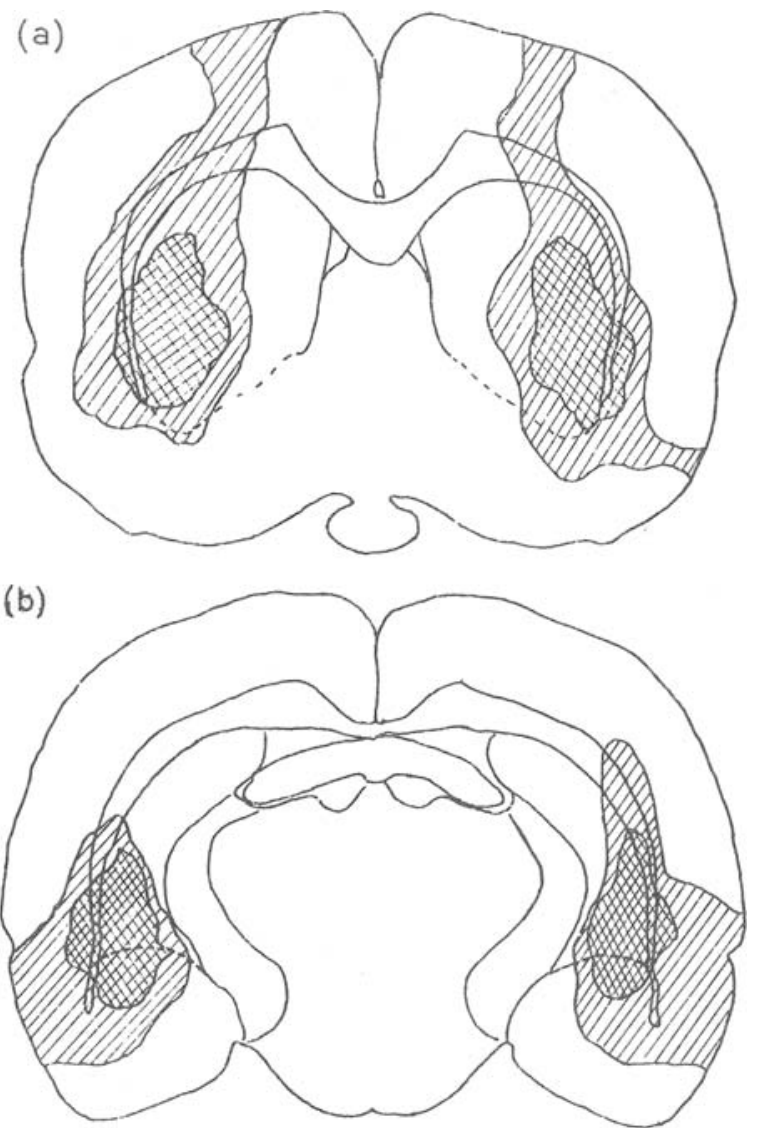

Figure 3. Representative damage in (a) anterior caudate operated animals and (b) posterior caudate operated animals. 


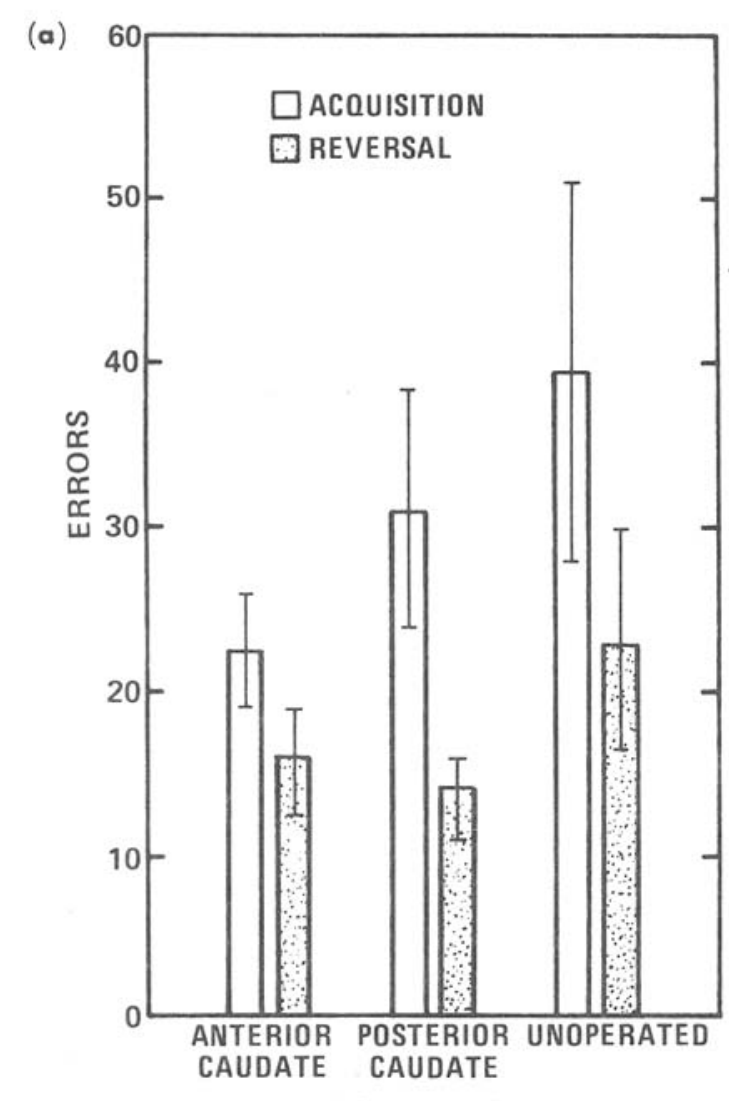

LESION GROUP

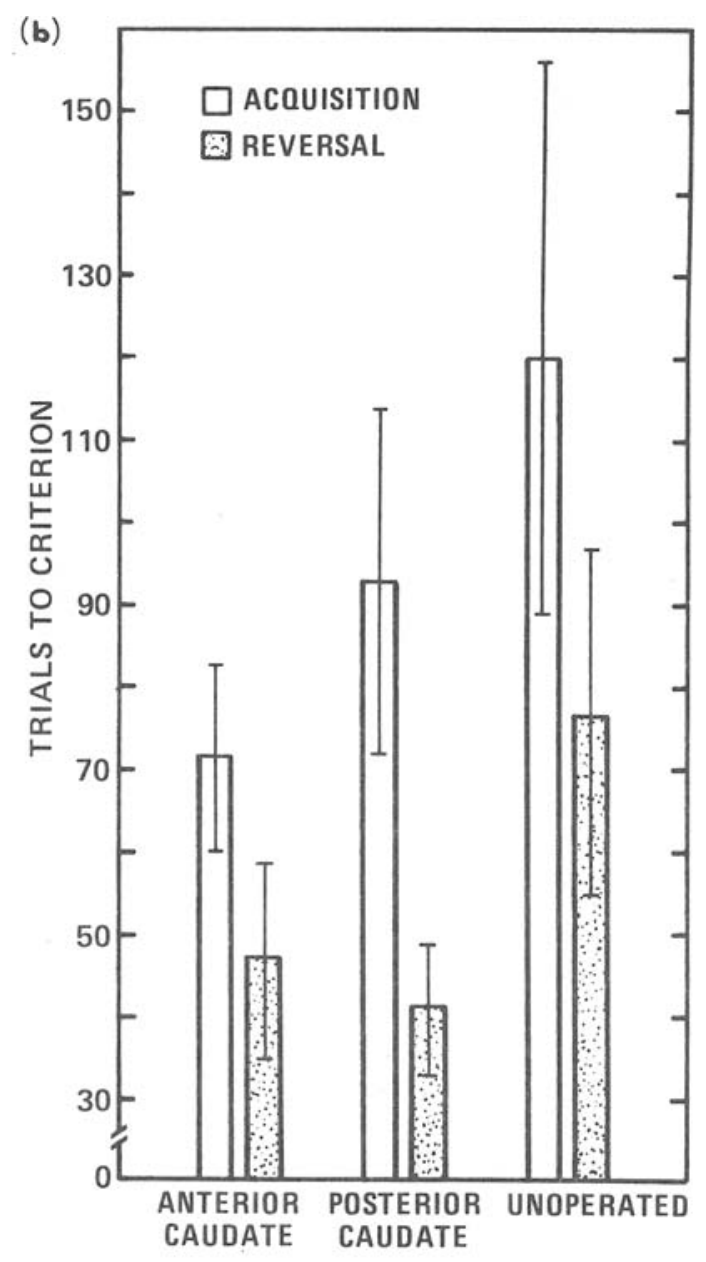

LESION GROUP

Figure 4. (a and b) Mean error and trials-to-criterion scores on acquisition.

consistently damaged in any specific group.

Acquisition. Figures $4 a$ and $4 b$ show the mean errors and trials to criterion for the groups. An analysis of variance of the acquisition error scores revealed a significant lesion effect $[F(2,63)=5.85, p<.005]$. A Newman-Keuls test of individual group comparisons showed that significantly more errors were made by the unoperated group (39.38) than by the anterior caudate group $(22.62 ; \mathrm{p}<.005)$. On this test, the posterior caudate group did not differ significantly from either the anterior caudate or the unoperated groups. Cue conditions did not produce a difference in acquisition error scores among the groups $[F(2,63)$ $<1.0]$. The statistical tests on the trials to criterion data gave the same results as those for errors. There were no significant differences in time scores among the groups $[F(2,63)=1.71, p>.19]$.

Reversal. Reversal data can be seen in Figures $4 a$ and $4 \mathrm{~b}$. The overall analysis of variance showed that reversal scores differed significantly from acquisition scores [for errors, $F(1,63)=37.31, p<.005$; and for trials-to-criterion, $\mathrm{F}(1,63)=32.77, \mathrm{p}<.005]$. On the reversal phase of the task, the groups differed significantly [for errors, $F(2,63)=5.82, p<$ .005 ; for trials, $F(2,63)=6.49, \mathrm{p}<.005]$. NewmanKeuls tests revealed that the unoperated animals made more errors and took more trials to reach criterion than did either posterior caudate $(p<.005)$ or anterior caudate $(\mathrm{p}<.02)$ animals.

Unlike acquisition, there were differences in reversal performance among the groups under the various cue conditions $[F(2,63)=4.81, p<.02]$. Newman-Keuls tests showed that all groups made fewer errors $(p<.02)$ under the tactile cue condition (13) than under visual cue (20) or no cue (21) conditions. In the visual cue condition, more errors were made $(\mathrm{p}<.05)$ by the unoperated animals (28) than by either the posterior caudates (14) or the anterior caudates (18). In the tactile cue condition, the only difference in error scores that approached significance by a NewmanKeuls test was that between the unoperated (19) and the posterior caudate $(9)$ animals $(p=.06)$. 
There were no significant differences in reversal response time scores among the various groups or cue conditions $[\mathrm{F}(2,63)<1.0]$.

\section{DISCUSSION}

The results of Experiment 1 support Potegal's (1972) hypothesis that the caudate nucleus is involved in spatial localization. Animals with caudate damage much more frequently used place rather than response cues in learning this task, which could be learned by using either. The performance of the rats with cortical lesions was similar to that reported by Pohl (1973) for his monkeys with lesions in the parietal cortex. Our cortical animals were response, rather than place, learners. Like Pohl's parietal monkeys, they used a body position referent rather than external spatial referents in learning this task.

Whether an animal was a place or response learner was not affected by overtraining. In an earlier study (Hicks, 1964), increased overtraining produced more response learners in normal rats.

Acquisition performance was not impaired by caudate lesions in either Experiment 1 or Experiment 2. Similar results on the acquisition of maze tasks by caudate and cortical rats have been reported by other investigators (Hannon \& Bader, 1974; Kirkby, 1969; Mikulas, 1966). The effects of caudate lesions on r reversal performance in our tasks is more complex. In Experiment 1, the caudate animals were impaired on reversal relative to their own acquisition performance but not relative to the reversal performance of the cortical or unoperated animals. Under the conditions of Experiment 1, the reversal from place to response (or response to place) is not the same as reversals in Experiment 2, which were east goalbox rewarded, not west box, or right turn rewarded, not left turn. The reversals used in Experiment 2 produced no deficit in our caudate animals, although it is typical of the types of reversals used in those experiments that do show impaired caudate reversal learning (Hannon \& Bader, 1974; Kirkby, 1969; Livesey \& Muter, 1976). This type of reversal could be called intradimensional, while a reversal from place to response, as in our Experiment 1, could be called extradimensional. Our caudate animals were impaired on extradimensional, but not on intradimensional, reversals. Our failure in Experiment 2 to find a deficit in caudate performance on this task, although contrary to some previous results (e.g., Kirkby, 1969), is consistent with a study by Thompson (cited in Schwartzbaum \& Donovick, 1968). Thompson found that caudate rats were not impaired in T-maze acquisition or reversal performance on a brightness discrimination (black and white side arms of the Tunit) or in spatial discrimination.

While the performance of our caudate animals was no different from that of unoperated animals in Ex- periment 1, it was improved over that of unoperated animals in Experiment 2. Our data from Experiment 1 led us to reason that differences in response time might be the basis of these results. In Experiment 1, both operated groups (cortical and caudate) were slower than unoperated animals in the time it took them to run a trial in the maze. Observations of locomotion in the maze and in an open field apparatus did not show any obvious differences in motor abilities between the operated and unoperated animals. But in the maze, unoperated rats nearly always went from startbox to goalbox without hesitation, while the operated rats, especially the caudates, stopped, reared, and retraced frequently. This slow and somewhat jerky progression of the operated animals did not produce more errors or more trials to criterion than did the smoother run of the unoperated animals. Frequent stops and starts along the maze path might have been expected to provide distractions that would impair performance. On the other hand, the slower traverse may have provided the operated animals with greater opportunity to explore and learn the maze, and, in this way, permitted them to maintain the accuracy of correct discriminations at the same level as their faster running, intact cohorts. Unfortunately, an inverse correlation between running time and errors or trials to criterion was not obtained uniformly across groups, although it did occur in some groups.

Kirkby's (1973) report of increased arousal and heightened reactivity in rats with caudate lesions provides another possible hypothesis for the lack of impairment in our caudate animals. The manner in which our caudate animals ran the maze is consistent with a hypothesis of increased arousal. Perhaps this mechanism allowed the caudate animals to use effectively the cues available in the maze tasks.

Differences between anterior dorsal and posterior caudate lesions were not found in our study. This is contrary to the report of Livesey and Muter (1976). Tactile cues uniformly improved the performance of all groups. Our unoperated animals, like those of Mikulas (1966), when compared with caudate animals, made more errors than either of the caudate groups in the presence of a visual cue.

The finding of no difference or improved performance on a T-maze task by the caudate animals in our two experiments could reasonably be interpreted as a true compensatory effect shown in the performance of animals with small caudate lesions. One might argue that the size of the cortical lesion was indeed too small to show a performance deficit, although the frequency of response learners in the cortical group indicates that their behavior is different from caudate or unoperated animals. Our caudate lesions, although subtotal and less than the $70 \%$ destruction claimed necessary to show behavioral effects in cats (Villablanca, 1975), are comparable to 
those that have produced performance deficits on reversal tasks in other studies (Hannon \& Bader, 1974; Kirkby, 1969; Mikulas, 1966). It is probable that the performance required on the T-maze tasks we use is different from that required in the tasks of those studies that report a deficit in rats with caudate lesions.

\section{REFERENCES}

Blodgett, H. C., \& McCutchan, K. Place versus response learning in the simple T-maze. Journal of Experimental Psychology, 1947, 37, 412-422.

Divac, I. Frontal lobe system and spatial reversal in the rat. Neuropsychologia, 1971, 9, 175-183.

Divac, I., Rosvold, H. E., \& Szwarcbart, M. K. Behavioral effects of selective ablation of the caudate nucleus. Journal of Comparative and Physiological Psychology, 1967, 63, 184-190.

HanNon, R., \& BADER, A. A comparison of frontal pole, anterior median and caudate nucleus lesions in the rat. Physiology \& Behavior, 1974, 13, 513-521.

Hicks, L. H. Effects of overtraining on acquisition and reversal of place and response learning. Psychological Reports, 1964, $15,459-462$.

KIRKBY, R. J. Caudate nucleus lesions and perseverative behavior. Physiology \& Behavior, 1969, 4, 451-454.

KIRKBY, R. J. Caudate nucleus and arousal in the rat. Journal of Comparative and Physiological Psychology, 1973, 85, 82-96.

KolB, B. Studies on the caudate-putamen and the dorsomedial thalamic nucleus of the rat: Implications for mammalian frontallobe functions. Physiology \& Behavior, 1977, 18, 237-244.

KriEG, W. J. S. Connections of the cerebral cortex. I. The albino rat. A topography of the cortical areas. Journal of Comparative Neurology, 1946, 84, 221-227.

Livesey, P. J., \& Muter, V. Functional differentiation within the neostriatum of the rat using electrical (blocking) stimulation during discrimination learning. Journal of Comparative and Physiological Psychology, 1976, 90, 203-211.

Mackintosh, N. J. Overtraining, transfer to proprioceptive control and position reversal. Quarterly Journal of Experimental Psychology, 1965, 17, 26-36.

Mikulas, W. L. Effects of lights at the choice point on spatial alternation and position learning by normal rats and rats with bilateral lesions of the caudate nucleus. Psychonomic Science, 1966, 5, 275-276.

Nfill, D. B., Bogigan, W. O., \& Grossman, S. P. Behavioral effects of amphetamine in rats with lesions in the corpus striatum. Journal of Comparative and Physiological Psychology, 1974, 86, 1019-1030.

Pelleginino, L. J., \& Cushman, A. J. A stereotaxic atlas of the rat brain. New York: Appleton-Century-Crofts, 1967.

Pонц, W. Dissociation of spatial discrimination deficits following frontal and parietal lesions in monkeys. Journal of Comparative and Physiological Psychology, 1973, 82, 227-239.

Potegal, M. Role of the caudate nucleus in spatial orientation of rats. Journal of Comparative and Physiological Psychology, 1969, 69, 756-764.

Potegal, M. The caudate nucleus egocentric localization system. Acta Neurobiologiae Experimentalis, 1972, 32, 479-494.

Restl., F. Discrimination of cues in mazes: A resolution of the place vs. response controversy. Psychological Review, 1957, 64, 217-228.

Ritchie, B. F., Aeschliman, B., \& Peirce, P. Studies in spatial learning VIII. Place performance and the acquisition of place dispositions. Journal of Comparative and Physiological Psychology, 1950, 43, 73-85.

Rosvold, H. E. The prefrontal cortex and caudate nucleus: A system for effecting correction in response mechanisms. In C. Rupp (Ed.), Mind as a tissue. New York: Harper \& Row, 1968.

Schwartzbaum, J. S., \& Donovick, P. J. Discrimination reversal and spatial alternation with septal and caudate dysfunction in rats. Journal of Comparative and Physiological Psychology, 1968, 65, 83-92.

Tolman, E. C., Ritchie, B. F., \& Kalish, D. Studies in spatial learning. V. Response learning vs. place learning by the noncorrection method. Journal of Experimental Psychology, 1947, 37, 285-292.

Villablanca, J. R., \& Marcus, R. J. Effects of caudate nuclei removal in cats. Comparison with effects of frontal cortex ablation. In N. A. Buchwald \& M. A. B. Brazier (Eds.), Brain-mechanisms in mental retardation. New York: Academic Press, 1975.

Winocur, G. Functional dissociation with the caudate nucleus of rats. Journal of Comparative and Physiological Psychology, 1974, 86, 432-439.

(Received for publication July 6, 1979; revision accepted June 2, 1980.) 